\section{Impacto orçamentário da incorporação do GeneXpert MTB/RIF para o diagnóstico da tuberculose pulmonar na perspectiva do Sistema Único de Saúde, Brasil, 2013-2017}

\section{Budget impact of the incorporation of GeneXpert MTB/RIF for diagnosis of pulmonary tuberculosis from the perspective of the Brazilian Unified National Health System, Brazil, 2013-2017}

Impacto presupuestario de la incorporación del GeneXpert MTB/RIF para el diagnóstico de la tuberculosis pulmonar desde la perspectiva del Sistema Único de Salud, Brasil, 2013-2017

\section{Resumo}

O objetivo do estudo foi estimar o impacto orçamentário do GeneXpert MTB/ RIF para o diagnóstico da tuberculose sob a perspectiva do Programa Nacional de Controle da Tuberculose, valendo-se de um modelo estático apoiado no método epidemiológico entre 2013 e 2017. Comparou-se um teste Xpert MTB/RIF com duas baciloscopias diagnósticas. Utilizaram-se dados epidemiológicos, populacionais, de custos, a taxa de câmbio e bases de dados do Sistema Único de Saúde. Foi realizada análise de sensibilidade por cenários. A incorporação do GeneXpert MTB/RIF demandaria um montante de R\$ 147 milhões em cinco anos e representaria um impacto de $23 \%$ a $26 \%$ nos dois primeiros anos, e de cerca de 11\% entre 2015 e 2017. Os resultados podem apoiar os gestores brasileiros e dos países latino-americanos no planejamento e gestão na sua decisão de incorporação da tecnologia.

Tuberculose; Reação em Cadeia da Polimerase; Diagnóstico; Custos e Análise de Custo

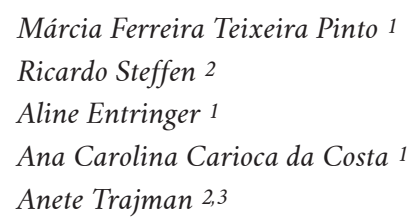

doi: 10.1590/0102-311X00214515

\author{
Correspondence \\ M. F. T. Pinto \\ Instituto Nacional de Saúde da Mulher, da Criança e do \\ Adolescente Fernandes Figueira, Fundação Oswaldo Cruz. \\ Av. Rui Barbosa 716, Rio de Janeiro, RJ 22250-020, Brasil. \\ mftpinto@gmail.com \\ 1 Instituto Nacional de Saúde da Mulher, da Criança e do \\ Adolescente Fernandes Figueira, Fundação Oswaldo Cruz, \\ Rio de Janeiro, Brasil. \\ 2 Faculdade de Medicina, Universidade Federal do \\ Rio de Janeiro, Rio de Janeiro, Brasil. \\ ${ }_{3}^{3}$ Montreal Chest Institute, McGill University, Montréal, \\ Canada.
}




\section{Introdução}

A tuberculose (TB) é um grave problema de saúde pública global. Estimativas indicam que em 2014, 9,6 milhões de indivíduos desenvolveram a doença, dos quais, 1,1 milhão era portador do HIV 1 . Na América Latina, ocorrem cerca de 268 mil casos novos de TB ao ano e 67\% estão na América do Sul 2. O Brasil está entre os 22 países com a maior carga global de TB e a cada ano são notificados aproximadamente 70 mil casos da doença 1 .

A TB acomete o pulmão na maioria dos casos e por isto seu mais frequente sintoma é a tosse. Todo indivíduo com mais de três semanas de tosse (chamado de sintomático respiratório), independentemente de ter ou não outros sintomas sugestivos da doença, deve ser submetido à investigação para TB. Em média, na população brasileira, estima-se que 3\% ou $4 \%$ dos sintomáticos respiratórios examinados sejam casos de TB bacilífera 3 . Outros sintomas sugestivos da doença são febre, perda de peso, sudorese noturna e fadiga. Indivíduos com esses sintomas e que apresentam tosse - independentemente da sua duração - também devem ser investigados para TB.

A dificuldade de acesso ao diagnóstico rápido da TB é um dos mais importantes obstáculos para o controle da doença. Especialmente em países de baixa e média renda, o diagnóstico se baseia na baciloscopia, devido às reduzidas exigências de infraestrutura para a sua realização e ao custo reduzido 1. No entanto, a acurácia do teste é baixa 4 , o que contribui para o atraso no diagnóstico e, por consequência, no aumento da mortalidade e morbidade, e na perpetuação da cadeia de transmissão 5

No Brasil e na América Latina, a baciloscopia ainda é muito utilizada, não apenas para o diagnóstico, como também para o controle do tratamento 2 . Entretanto, diante das limitações desse teste convencional para o diagnóstico da TB, novas tecnologias surgiram, como o Xpert MTB/RIF, teste molecular rápido realizado no sistema GeneXpert MTB/RIF, para a detecção do Mycobacterium tuberculosis e da resistência à rifampicina 6,7. O Xpert MTB/RIF é particularmente útil nos países com altas taxas de TB-multirresistente (TB-MDR, resistente à rifampicina e isoniazida), como o Peru, responsável por 32\% dos casos na região 1,2. Desde a sua recomendação pela Organização Mundial da Saúde (OMS) em 2010 8, vários sistemas de saúde já o incorporaram, inclusive a maioria dos países latino-americanos já adquiriu o GeneXpert MTB/RIF para o diagnóstico da TB 9. No Brasil, o teste foi aprovado pela Comissão Nacional de Incorporação de Tecnologias no SUS (CONITEC) em 201310 e a incorporação no Sistema Único de Saúde (SUS) ocorreu no mesmo ano. O país é o principal mercado consumidor de cartuchos entre os países sul-americanos ${ }^{9}$. Neste trabalho, quando se menciona GeneXpert MTB/RIF, trata-se do sistema como um todo, que inclui máquinas, computador e nobreak. Já o teste Xpert MTB/RIF refere-se ao cartucho, insumos e reagentes.

O potencial do Xpert MTB/RIF no aumento da taxa de notificação de casos e na redução da incidência, morbidade e mortalidade da TB tem sido considerado nas avaliações econômicas, que incluem a análise de impacto orçamentário. Estudos sugerem que o Xpert MTB/RIF é custo-efetivo em diferentes cenários 11,12,13,14,15. Também no Brasil, um país de média renda, o teste para uma amostra de escarro se mostrou mais custo-efetivo 15 do que a baciloscopia de duas amostras diagnósticas na perspectiva do SUS. Embora uma tecnologia seja custo-efetiva, pode haver insuficiência de recursos para a sua incorporação ${ }^{16}$, principalmente quando há dependência de insumos importados que sofrem variação de preços devido à taxa de câmbio, uma variável dinâmica e sensível ao cenário econômico dos países. Nesse contexto, a análise de impacto orçamentário contribui para que o orçamento destinado à incorporação, expansão da oferta e garantia da operacionalização eficiente da nova tecnologia possa ser garantido ao longo de um horizonte temporal e que seja acessível e sustentável para o sistema de saúde.

Este trabalho teve como objetivo realizar uma análise de impacto orçamentário do GeneXpert MTB/RIF entre 2013 e 2017, sob a perspectiva do Programa Nacional de Controle da Tuberculose (PNCT) do Ministério da Saúde, com base em projeções de notificação de casos de TB diagnosticados pelo Xpert MTB/RIF como substituto de duas baciloscopias diagnósticas de primeira amostra, da quantidade necessária de testes para diagnosticar um caso de TB e do cenário econômico de apreciação da taxa de câmbio no Brasil ao longo dos últimos anos. No contexto regional, esta análise de impacto orçamentário pretende oferecer subsídios a outros países da América Latina que cogitem incorporar o GeneXpert MTB/RIF ou ampliar a sua incorporação naqueles que já o têm. 


\section{Materiais e métodos}

\section{Tecnologia}

O GeneXpert MTB/RIF é um equipamento composto por módulos com cartuchos individuais de uso único onde se coloca uma alíquota de amostra para a detecção de moléculas por reação em cadeia da polimerase. O sistema pode fazer diagnóstico rápido de várias doenças infecciosas ou oncológicas, mas no Brasil está sendo usado para a detecção da TB utilizando o cartucho de Xpert MTB/RIF. O equipamento, que conta com computador e software próprios, fornece o resultado em aproximadamente 105 minutos, mas requer rede elétrica estável para o seu funcionamento e acondicionamento dos cartuchos em ambiente refrigerado entre $2^{\circ}$ e $28^{\circ} \mathrm{C}$. Pode ser operado no mesmo espaço físico onde é realizada a baciloscopia e não exige condições especiais de biossegurança 17. Dados de acurácia do novo teste demonstraram uma sensibilidade de 89\% (IC95\%: 85\%; 92\%) e especificidade de 99\% (IC95\%: 98\%; 99\%) em substituição à baciloscopia de primeira amostra ${ }^{7}$ para a detecção de TB. Material insuficiente (escarro) ou inadequado (presença de saliva) são problemas observados durante a sua realização 18,19 .

\section{Desenho do estudo}

Trata-se de uma análise de impacto orçamentário baseada em um modelo estático que utilizou uma calculadora de custos desenvolvida em planilha eletrônica determinística elaborada em Excel 2010 (Microsoft Corp., Estados Unidos) e que incorporou, para o Xpert MTB/RIF e a baciloscopia, parâmetros populacionais, epidemiológicos (casos notificados, taxa de notificação e de abandono de tratamento), pressupostos associados à realização dos testes por sintomáticos respiratórios e custo do diagnóstico e tratamento da TB. A perspectiva da análise é a do PNCT, responsável pelo financiamento do diagnóstico e tratamento da TB no SUS e pelo planejamento e organização da implantação da nova tecnologia.

O horizonte temporal é de cinco anos, de 2013 a 2017, pois acredita-se que neste período a tecnologia estará bem estabelecida no SUS e permite ao PNCT conhecer o impacto orçamentário anual especialmente a partir da projeção de notificações. A análise foi dividida em dois momentos devido às especificidades de incorporação do novo teste: o primeiro correspondeu aos anos de 2013 e 2014, pois a baciloscopia ainda não havia sido totalmente substituída 20, e o segundo de 2015 a 2017, quando os dois testes são excludentes, ou seja, já há substituição da baciloscopia diagnóstica pelo Xpert MTB/ RIF nos municípios selecionados pelo PNCT para a sua incorporação.

A compra e instalação do sistema GeneXpert MTB/RIF pelo PNCT iniciou em 2013. A implantação da Rede de Teste Rápido para TB (RTR-TB) teve início em julho de 201420 e incluiu 94 municípios, onde são notificados cerca de 60\% dos casos no Brasil, conforme o Sistema de Informação de Agravos de Notificação (SINAN; http://www2.datasus.gov.br/DATASUS/index.php?a rea $=0203 \& i d=31009407$ ).

\section{População de interesse e estimativa de notificação de casos de TB}

A população elegível para o novo teste é a mesma que é elegível para a baciloscopia, ou seja, os sintomáticos respiratórios e os com suspeita de TB. Foi utilizado o método epidemiológico 21 com base no número de indivíduos testados por baciloscopia e, se positivos, notificados no SINAN. Estimou-se a população geral e os casos notificados para 2015, 2016 e 2017 para cada município selecionado. Para 2013 e 2014, os dados de notificação já estavam disponibilizados no SINAN. Para a estimativa da população geral aplicou-se um modelo de regressão linear simples, utilizando-se dados de censos e contagens populacionais (Instituto Brasileiro de Geografia e Estatística; http://www.ibge.gov.br). Para os casos notificados de TB de 2015 a 2017, adotou-se um modelo de regressão de Poisson e a população de cada município como offset 22 . Ressalta-se que os gráficos de autocorrelação e autocorrelação parcial não revelaram indícios de dependência ao longo do tempo para nenhum dos municípios analisados. As análises estatísticas tiveram como referência um nível de significância de $5 \%$ e foram realizadas no software R, versão 3.1.3 (R Development Core Team. http://www.R-project.org). 


\section{Cenário de referência}

O cenário de referência da análise de impacto orçamentário foi o ano de 2013, considerado aqui como o ano inicial de aquisição dos equipamentos pelo PNCT a partir da incorporação do Xpert MTB/RIF na rede de serviços de saúde de dois municípios (Rio de Janeiro e Manaus, Amazonas) em um estudo piloto de implantação 11 . Naquele ano, a baciloscopia era o teste de rotina. Utilizaram-se como parâmetros para os cálculos do impacto orçamentário os critérios do PNCT para a seleção dos municípios: (i) municípios que notificaram mais de 200 casos novos de TB em 2011 e que tinham um laboratório com estrutura física e de biossegurança equivalente à necessária para a realização da baciloscopia; (ii) capitais estaduais que não notificaram mais de 200 casos novos em 2011, mas que são referência para o diagnóstico e tratamento da TB nos seus estados; (iii) municípios onde há presídios ou com população indígena que notificaram pelo menos 50 casos em 2011, critério utilizado pela alta taxa de TB-MDR nos presídios e pela dificuldade do diagnóstico nas populações indígenas; e (iv) Laboratórios Centrais de Saúde Pública, que são coordenadores da rede estadual de laboratórios e responsáveis pelos treinamentos e controle de qualidade dos exames realizados. Ademais, a nova tecnologia gera um incremento da taxa de notificação de TB em 15\% (IC95\%: -6\%; 37\%) que foi incluída no modelo ${ }^{11}$.

Foram adotados os seguintes pressupostos para o cenário de referência: (i) realização de cinco testes Xpert MTB/RIF ou baciloscopia para a detecção de um caso de TB. Esse pressuposto foi necessário devido à ausência de dados dos sintomáticos respiratórios que são submetidos aos testes e do quantitativo de testes utilizado para cada paciente ser diagnosticado; (ii) o número de casos notificados entre 2014 e 2017 foi corrigido pelo aumento da taxa de notificação em 15\% 11. Não houve correção em 2013 pois a baciloscopia era o teste de rotina; (iii) 100\% dos casos notificados iniciariam o tratamento; (iv) ajuste do total de casos notificados baseando-se na taxa de abandono do tratamento de $11 \%$ 23; e (v) para a taxa de difusão da tecnologia no SUS em 2013, assumiu-se que 100\% dos casos notificados foram diagnosticados com a baciloscopia, em 2014, 50\% foram diagnosticados com Xpert MTB/RIF pois o teste começou a ser realizado nos laboratórios na metade do ano e, nos anos seguintes, 100\% dos diagnósticos seriam realizados com a nova tecnologia.

\section{Identificação, quantificação e valoração dos recursos e insumos de saúde}

\section{- Custos do diagnóstico e tratamento}

O custo individual esperado do diagnóstico com a baciloscopia para o cenário de referência foi de R\$ 100,25 e com o Xpert MTB/RIF de R\$ 167,41 por caso notificado. O custo individual esperado do tratamento completo por caso notificado, para ambos os testes, foi de $\mathrm{R} \$ 289,51$. Esses custos esperados referem-se aos resultados de estudo prévio que utilizou um modelo de análise de decisão para estimar a razão de custo-efetividade da nova tecnologia 15. Considerou-se que o custo do tratamento do paciente que não o completou seria de R\$ 189,51 para uma taxa de $11 \%$ de abandono 23 . Adotou-se como pressuposto que o custo unitário da baciloscopia não alteraria no período da análise (Tabela 1). A identificação e quantificação dos insumos de saúde foram baseadas nas diretrizes do PNCT, os itens de custos do diagnóstico foram consultas, testes (duas baciloscopias ou um Xpert MTB/RIF) e exames laboratoriais.

Para o tratamento, foram considerados consultas, exames laboratoriais, medicamentos e hospitalização, e também os eventos adversos. O custo unitário para produzir cada teste Xpert MTB/RIF foi baseado na literatura valendo-se da técnica de microcusto e incluiu o custo de aquisição, manutenção e depreciação dos equipamentos do sistema Gene Xpert MTB/RIF, de aquisição de reagentes, insumos e do cartucho, recursos humanos 24 e desperdício do cartucho (6,6\% devido à amostra ter volume insuficiente ou ser inadequada) 11 . O custo do cartucho foi de $\mathrm{R} \$ 17,46$, valor negociado pelo PNCT com a Foundation for Innovative New Diagnostics (Genebra, Suíça), que tem definida uma lista de países para os quais pode ser adquirido pelo setor público a um preço fixo de US\$ 9,98. O Brasil, bem como a totalidade dos países da América Latina, faz parte dessa lista 25.

O cálculo do custo total de aquisição das máquinas teve como base um valor unitário de $\mathrm{R} \$ 35.875,00$, que se refere ao preço de compra para o estudo piloto de implantação da nova tecnologia 11,24. No pri- 


\section{Tabela 1}

Parâmetros populacionais, epidemiológicos, de produção de testes e de custos utilizados no cenário de referência da análise de impacto orçamentário do GeneXpert MTB/RIF.

\begin{tabular}{|c|c|c|c|c|c|c|c|c|c|c|c|}
\hline \multirow[t]{2}{*}{ Parâmetros } & \multicolumn{2}{|c|}{2013 * } & \multicolumn{2}{|c|}{2014 ** } & \multicolumn{2}{|c|}{2015} & \multicolumn{2}{|c|}{2016} & \multicolumn{2}{|c|}{2017} & \multirow[t]{2}{*}{ Fonte } \\
\hline & $\begin{array}{l}\text { Gene- } \\
\text { Xpert }\end{array}$ & $\begin{array}{l}\text { Bacilos- } \\
\text { copia }\end{array}$ & $\begin{array}{l}\text { Gene- } \\
\text { Xpert }\end{array}$ & $\begin{array}{l}\text { Bacilos- } \\
\text { copia }\end{array}$ & $\begin{array}{l}\text { Gene- } \\
\text { Xpert }\end{array}$ & $\begin{array}{l}\text { Bacilos- } \\
\text { copia }\end{array}$ & $\begin{array}{l}\text { Gene- } \\
\text { Xpert }\end{array}$ & $\begin{array}{l}\text { Bacilos- } \\
\text { copia }\end{array}$ & $\begin{array}{l}\text { Gene- } \\
\text { Xpert }\end{array}$ & $\begin{array}{l}\text { Bacilos- } \\
\text { copia }\end{array}$ & \\
\hline \multicolumn{12}{|l|}{ Populacionais } \\
\hline $\begin{array}{l}\text { População dos } \\
\text { municípios } \\
\text { selecionados para } \\
\text { incorporar o sistema } \\
\text { GeneXpert }\end{array}$ & \multicolumn{2}{|c|}{72.414 .608} & \multicolumn{2}{|c|}{73.045 .249} & \multicolumn{2}{|c|}{73.269 .801} & \multicolumn{2}{|c|}{73.992 .745} & \multicolumn{2}{|c|}{74.715 .680} & IBGE \\
\hline \multicolumn{12}{|l|}{ Epidemiológicos } \\
\hline $\begin{array}{l}\text { Casos notificados } \\
\text { (aumento da taxa de } \\
\text { notificação de TB em } \\
15 \% \text { com Xpert) }\end{array}$ & 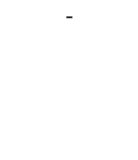 & 43.168 & 27.410 & 17.617 & 49.429 & 42.982 & 49.732 & 43.245 & 50.092 & 43.558 & $\begin{array}{l}\text { Durovni et } \\
\text { al. 11; SINAN }\end{array}$ \\
\hline \multicolumn{12}{|l|}{ Produção } \\
\hline Testes/ano *** & - & 215.840 & 137.051 & 88.085 & 247.147 & 214.910 & 248.659 & 216.225 & 250.459 & 217.790 & Pressuposto \\
\hline \multicolumn{12}{|l|}{ Custos (R\$) } \\
\hline Sistema GeneXpert & 35.875 & - & 35.875 & - & - & - & - & - & - & - & Pinto et al. 24 \\
\hline $\begin{array}{l}\text { Quantidade de } \\
\text { máquinas }\end{array}$ & 116 & - & 42 & - & - & - & - & - & - & - & $\begin{array}{l}\text { Ministério da } \\
\text { Saúde } 27\end{array}$ \\
\hline $\begin{array}{l}\text { Custo total dos } \\
\text { equipamentos }\end{array}$ & 4.161 .500 & - & 1.506 .750 & - & - & - & - & - & - & - & $\begin{array}{l}\text { Ministério da } \\
\text { Saúde } 23\end{array}$ \\
\hline $\begin{array}{l}\text { Diagnóstico por caso } \\
\text { notificado }\end{array}$ & - & 100,25 & 167,41 & 100,25 & 167,41 & 100,25 & 167,41 & 100,25 & 167,41 & 100,25 & Pinto et al. 15 \\
\hline $\begin{array}{l}\text { Tratamento por caso } \\
\text { notificado }\end{array}$ & \multicolumn{2}{|c|}{289,51} & \multicolumn{2}{|c|}{289,51} & \multicolumn{2}{|c|}{289,51} & \multicolumn{2}{|c|}{289,51} & \multicolumn{2}{|c|}{289,51} & Pinto et al. 15 \\
\hline $\begin{array}{l}\text { Tratamento } \\
\text { (ajustado pela taxa } \\
\text { de abandono) }\end{array}$ & \multicolumn{2}{|c|}{189,51} & \multicolumn{2}{|c|}{189,51} & \multicolumn{2}{|c|}{189,51} & \multicolumn{2}{|c|}{189,51} & \multicolumn{2}{|c|}{189,51} & $\begin{array}{l}\text { Ministério da } \\
\text { Saúde } 23\end{array}$ \\
\hline
\end{tabular}

IBGE: Instituto Brasileiro de Geografia e Estatística; SINAN: Sistema de Informação de Agravos de Notificação.

* Inclui somente o custo do sistema GeneXpert MTB/RIF;

** Considerou-se que $50 \%$ dos casos seriam notificados pelo Xpert MTB/RIF devido à sua implantação na metade de 2014;

*** Produção de cinco testes para a notificação de um caso de tuberculose.

meiro ano desta análise (2013), somente foram contabilizados os custos com a compra do equipamento (Tabela 1).

\section{- Cálculo do impacto orçamentário}

O custo total do GeneXpert MTB/RIF foi calculado pela multiplicação do custo unitário esperado pelo número de indivíduos com indicação de diagnóstico, com ajustes pela demanda de testes, taxa de notificação e abandono. Desse resultado, subtraiu-se o custo total das baciloscopias de duas amostras para fins de diagnóstico, que também foi calculado pela multiplicação do custo unitário pelo número de indivíduos com a mesma indicação. Excepcionalmente, devido à taxa de difusão da nova tecnologia, foi considerado que em 2013 e até a metade de 2014 haveria um custo adicional para o PNCT. Nesse período, o processo de conformação da RTR-TB foi simultâneo à realização de baciloscopia nos municípios. Essa especificidade da incorporação requereu que os custos fossem somados e não subtraídos. Em termos proporcionais, comparou-se o resultado do impacto orçamentário com o 
orçamento anual do PNCT 1 entre 2013 e 2017. Em 2016 e 2017, devido à indisponibilidade de informações, foi mantido o mesmo orçamento de 2015. Esses dados estão informados em dólares ${ }^{1}$ e foram convertidos para reais com base no dólar médio anual dos anos de 2013 ( R\$ 2,28), 2014 ( $\mathrm{R} \$ 2,41)$ e 2015 (R\$ 3,64). Para 2016 e 2017, utilizou-se R\$ 3,66 e R\$ 3,50, respectivamente. O ajuste inflacionário não foi considerado e os valores são apresentados em reais de 2013.

\section{Análise de sensibilidade}

Análises por cenários foram realizadas devido à possibilidade dos seguintes parâmetros e pressupostos gerarem incertezas nos resultados:

I) Variação da taxa de câmbio e influência no valor do cartucho: devido à apreciação do dólar frente ao real, para 2015 e 2016, projetou-se uma taxa de câmbio esperada pelos agentes financeiros de US\$ $=\mathrm{R} \$ 3,64$ e de US\$ $=\mathrm{R} \$ 3,66$, respectivamente 26. Para 2017, adotou-se como pressuposto a taxa de câmbio de US\$ = R\$ 3,50. Assumiu-se que em 2013 e 2014, o valor do cartucho não mudaria devido à aquisição prévia pelo PNCT.

II) Aumento da realização de testes Xpert MTB/RIF para a detecção de um caso de TB positivo: foi assumida a realização de dois testes (melhor cenário) e 11 testes (pior cenário) para detectar um caso, conforme dados de consumo mensal da RTR-TB 27 na tentativa de identificar a utilização e seu impacto no orçamento.

III) Variação da taxa de notificação de casos de TB: o Xpert MTB/RIF aumenta a notificação em $15 \%$, o que impacta diretamente no aumento da quantidade de tratamentos de TB. Como o impacto orçamentário é muito sensível a essa variável, utilizamos uma variação entre $-6 \%$ e $37 \%$ 11. A taxa de notificação da baciloscopia não foi variada.

\section{Considerações éticas}

O presente estudo foi aprovado pela Comissão Nacional de Ética em Pesquisa (CONEP) (protocolo no 493/2011), pelo Comitê de Ética em Pesquisa (CEP) da Secretaria Municipal de Saúde e Defesa Civil do Rio de Janeiro (SMSDC/RJ; protocolo no 445A/11) e pelo CEP da Fundação de Medicina Tropical de Manaus (protocolo FMT/HDV, 24 de novembro de 2011).

\section{Resultados}

O modelo foi revisado e testado após a sua conclusão, a fim de identificar possíveis erros relacionados com a incorporação dos dados e a sintaxe da programação no software utilizado. Essa validação interna garantiu que os cálculos matemáticos estavam exatos e consistentes com todos os parâmetros e pressupostos estabelecidos pelo modelo.

Os resultados do cenário de referência indicam que entre 2013 e 2017, o aumento da notificação de casos de TB seria de aproximadamente 12\% (Figura 1). A incorporação do GeneXpert MTB/RIF demandaria um montante de $\mathrm{R} \$ 147$ milhões em cinco anos para uma taxa de difusão de 100\% a partir de 2015 (Tabela 2). Em 2013, os diagnósticos realizados por baciloscopia ainda eram a rotina nos laboratórios. O custo total foi de R\$ 38 milhões, ou seja, o somatório dos custos das duas tecnologias, sendo que para o GeneXpert MTB/RIF foi considerada apenas a aquisição das máquinas. Em termos relativos, correspondeu a 23\% do orçamento do PNCT (Tabela 2). No segundo ano de incorporação (2014), o Xpert MTB/RIF foi utilizado apenas a partir da metade do ano, por isto houve um menor número de diagnósticos (137.051) e casos notificados (27.410) (Tabela 1). O impacto seria de R 44 milhões ou 26\% do orçamento do PNCT (Tabela 2). Em 2015, momento de incorporação plena da nova tecnologia, observou-se que o custo do diagnóstico e tratamento seria de $\mathrm{R} \$ 55$ milhões e o impacto no orçamento teria um incremento de R\$ 21,6 milhões em comparação com a baciloscopia (Tabela 2). Para 2016 e 2017, esse montante não variaria sobremaneira, mantendo-se entre $R \$ 21,8$ e 21,9 milhões e 11\% do orçamento do PNCT (Tabela 2).

Na análise de sensibilidade por cenários, no período de 2015 e 2017, o aumento da taxa de notificação em $6 \%$ reduziria o impacto orçamentário entre $46 \%-49 \%$, porém mais que dobraria se este 
Figura 1

Casos notificados de tuberculose entre 2002 a 2014, com projeção do número de notificações de 2015 a 2017 , Brasil.

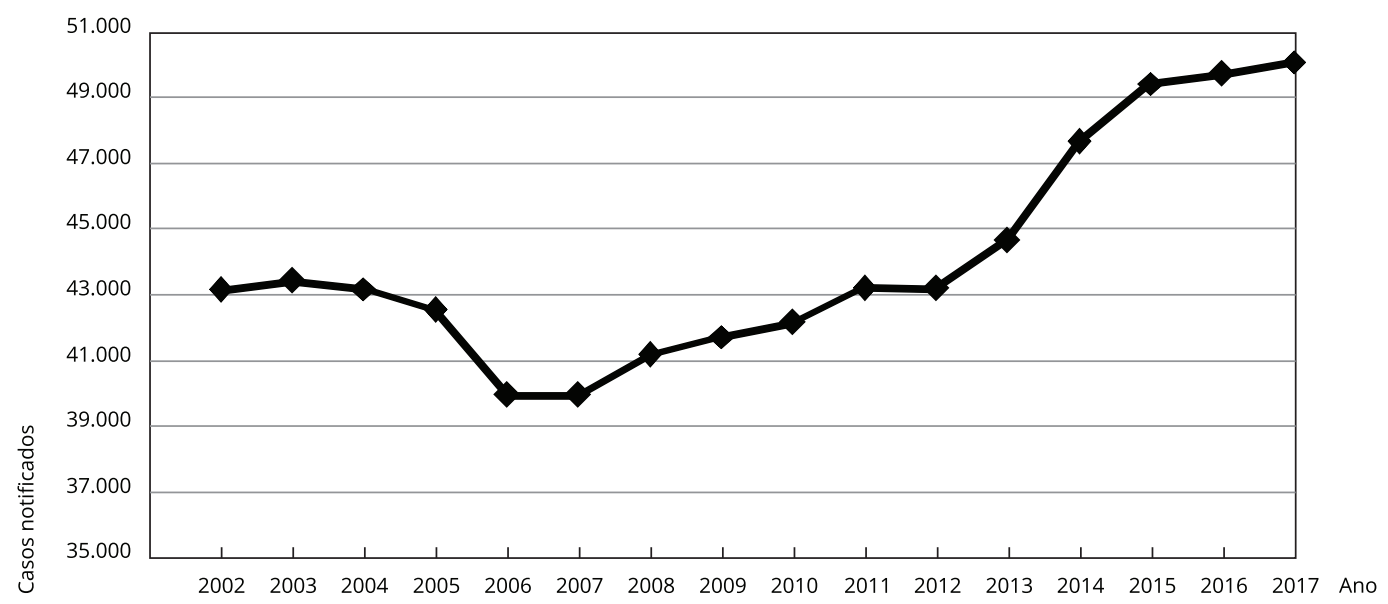

aumento fosse de $37 \%$ em relação ao cenário de referência. Em termos proporcionais ao orçamento do PNCT, o menor incremento da taxa seria responsável por 7\%-8\%, e o maior por $27 \%-28 \%$. Ao se variar a produção, o melhor cenário (dois testes) apontou uma redução do impacto superior a 100\% entre 2015 e 2017, mas para o pior cenário (11 testes) há um incremento de cerca de 55\% quando comparado com o cenário de referência para o mesmo período. Na comparação com o orçamento do PNCT, o melhor cenário seria responsável por 5\% e no pior cenário este valor mais que triplicaria, perfazendo $17 \%-18 \%$ (Tabela 3 ).

\section{Discussão}

A incorporação do GeneXpert MTB/RIF está em curso desde 2013, por intermédio da constituição da RTR-TB no SUS. Apesar disso, esta análise de impacto orçamentário teve a intenção de contribuir com o processo de planejamento e gestão da incorporação até 2017, e de servir como base para a reflexão de outros países que estejam cogitando incorporá-lo ou que desejam ampliar a sua oferta no sistema de saúde. Estimativas para além de 2017 elevariam a incerteza dos cálculos, especialmente devido à apreciação ou depreciação do dólar frente ao real, cujo comportamento depende de estimativas dos agentes econômicos que podem alterar com frequência 26 . A tomada de decisão apoiada em avaliação econômica é uma realidade em diversos países e, no Brasil, a realização de estudos desta natureza é consubstanciada principalmente pela necessidade de apresentarem evidências para a incorporação de tecnologias no âmbito do SUS 28. Pressupõe incluir não somente dados clínicos e epidemiológicos, mas também o comportamento de indicadores econômicos para predizer possíveis mudanças de custos no médio prazo.

O impacto orçamentário em termos relativos ao orçamento do PNCT se manteria estável entre 2015 e 2017, em 11\%-12\%, e em termos absolutos observou-se um aumento pequeno, embora gradual de R\$ 21,6 milhões em 2015 para 21,9 milhões em 2017. Tecnologias baseadas em equipamentos caracterizam-se por um investimento inicial elevado no momento da incorporação, mas que reduzem ao longo dos anos. Por isso, é necessário acompanhar o comportamento da taxa de câmbio quando se incorporam tecnologias cujos preços estão atrelados à moeda estrangeira. O Xpert MTB/RIF no Brasil tem como principal direcionador de custo o cartucho 24 e, portanto, poderá ocorrer um aumento 


\section{Tabela 2}

Impacto orçamentário anual do GeneXpert MTB/RIF para o cenário de referência, 2013 a 2017 (em Reais de 2013).

\begin{tabular}{|c|c|c|c|c|c|c|c|c|c|c|}
\hline \multirow[t]{2}{*}{ Custos (R\%) } & \multicolumn{2}{|c|}{2013} & \multicolumn{2}{|c|}{2014} & \multicolumn{2}{|c|}{2015} & \multicolumn{2}{|c|}{2016} & \multicolumn{2}{|c|}{2017} \\
\hline & $\begin{array}{l}\text { Gene- } \\
\text { Xpert }\end{array}$ & $\begin{array}{l}\text { Bacilos- } \\
\text { copia }\end{array}$ & $\begin{array}{l}\text { Gene- } \\
\text { Xpert }\end{array}$ & $\begin{array}{l}\text { Bacilos- } \\
\text { copia }\end{array}$ & $\begin{array}{l}\text { Gene- } \\
\text { Xpert }\end{array}$ & $\begin{array}{l}\text { Bacilos- } \\
\text { copia }\end{array}$ & $\begin{array}{l}\text { Gene- } \\
\text { Xpert }\end{array}$ & $\begin{array}{l}\text { Bacilos- } \\
\text { copia }\end{array}$ & $\begin{array}{l}\text { Gene- } \\
\text { Xpert }\end{array}$ & $\begin{array}{l}\text { Bacilos- } \\
\text { copia }\end{array}$ \\
\hline $\begin{array}{l}\text { Custo total do } \\
\text { diagnóstico * }(A)\end{array}$ & - & 21.637 .960 & 22.943 .750 & 8.830 .521 & 41.374 .796 & 21.544 .728 & 41.627 .961 & 21.676 .556 & 41.929 .257 & 21.833 .448 \\
\hline $\begin{array}{l}\text { Custo total do } \\
\text { tratamento ** }\end{array}$ & - & 12.497 .568 & 7.935 .541 & 5.100 .298 & 14.310 .277 & 12.443 .719 & 14.397 .839 & 12.519 .860 & 14.502 .048 & 12.610 .477 \\
\hline $\begin{array}{l}\text { Custo total do } \\
\text { tratamento } \\
\text { (ajustado pela taxa } \\
\text { de abandono) ** (B) }\end{array}$ & - & 12.022 .720 & 7.634 .029 & 4.906 .511 & 13.766 .554 & 11.970 .917 & 13.850 .790 & 12.044 .165 & 13.951 .039 & 12.131 .339 \\
\hline $\begin{array}{l}\text { Custo total de cada } \\
\text { tecnologia }(A+B)\end{array}$ & 4.161 .500 & 33.660 .680 & 30.577 .778 & 13.737 .032 & 55.141 .350 & 33.515 .644 & 55.478 .751 & 33.720 .721 & 55.880 .297 & 33.964 .786 \\
\hline $\begin{array}{l}\text { Custo total } \\
\text { para o PNCT de } \\
\text { todos os casos } \\
\text { diagnosticados e } \\
\text { tratados (GeneXpert } \\
\text { MTB/RIF + } \\
\text { baciloscopia) }\end{array}$ & \multicolumn{2}{|c|}{37.822 .180} & \multicolumn{2}{|c|}{44.314 .810} & \multicolumn{2}{|c|}{-} & \multicolumn{2}{|c|}{ - } & \multicolumn{2}{|c|}{-} \\
\hline $\begin{array}{l}\text { Impacto } \\
\text { orçamentário } \\
\text { (GeneXpert MTB/RIF } \\
\text { + baciloscopia) }\end{array}$ & \multicolumn{2}{|r|}{-} & \multicolumn{2}{|c|}{-} & \multicolumn{2}{|c|}{21.625 .706} & \multicolumn{2}{|c|}{21.758 .030} & \multicolumn{2}{|c|}{21.915 .511} \\
\hline $\begin{array}{l}\text { Orçamento do } \\
\text { PNCT (em milhões) } \\
\text { e percentual do } \\
\text { orçamento *** }\end{array}$ & \multicolumn{2}{|c|}{$166(23 \%)$} & \multicolumn{2}{|c|}{$166(26 \%)$} & \multicolumn{2}{|c|}{$200(11 \%)$} & \multicolumn{2}{|c|}{$201(11 \%)$} & \multicolumn{2}{|c|}{$193(11 \%)$} \\
\hline
\end{tabular}

PNCT: Programa Nacional de Controle da Tuberculose.

* Custo total do diagnóstico refere-se ao número de testes realizados ao ano multiplicado pelo custo do diagnóstico por caso notificado;

** Custo total do tratamento refere-se ao número de casos notificados ao ano multiplicado pelo custo do tratamento por caso notificado, ajustado pela taxa de abandono:

*** Valor do orçamento anual em dólar do PNCT convertido em Reais.

do custo do diagnóstico com a valorização cambial, a não ser que ocorram negociações para fixar o preço em real. A taxa de notificação também é relevante na análise, pois quanto mais acurado o teste, maior o custo do tratamento da TB e, por consequência, maior o impacto orçamentário. Essa tendência deverá ser acompanhada em conjunto com o custo do tratamento.

Também é necessário garantir a eficiência do processo de realização do teste para a redução dos custos. O GeneXpert MTB/RIF tem capacidade de produção entre 16 e 20 testes diários para regimes de trabalho de dois e três turnos de seis horas, respectivamente. Em 2014, o PNCT recomendou a utilização da tecnologia para crianças menores de dez anos e para amostras de exames extrapulmonares, como líquor e amostras de gânglios e outros tecidos 29. Esse aumento de demanda poderá contribuir para a ampliação da capacidade da oferta e com a racionalização dos custos. Mas especificidades intrínsecas do teste, como material insuficiente (escarro) ou inadequado (presença de saliva), e do sistema de saúde (obstáculos logísticos) podem influenciar a eficiência na realização do diagnóstico de TB. Além do aumento da demanda, outros componentes deverão ser operacionalizados para que a rede funcione eficientemente e de maneira contínua, tais como equipe técnica disponível a fim de garantir a manutenção preventiva e corretiva em tempo hábil, rede de energia elétrica estável, logís- 
Análise de sensibilidade por cenários relativos ao impacto orçamentário do GeneXpert MTB/RIF, 2015 a 2017.

\begin{tabular}{|c|c|c|c|c|c|c|c|}
\hline & \multicolumn{2}{|c|}{2015} & \multicolumn{2}{|c|}{2016} & \multicolumn{2}{|c|}{2017} & \multirow[t]{2}{*}{ Fonte } \\
\hline & GeneXpert & Baciloscopia & GeneXpert & Baciloscopia & GeneXpert & Baciloscopia & \\
\hline \multicolumn{8}{|l|}{ Notificação * } \\
\hline Casos notificados de TB & 42.982 & - & 43.245 & - & 43.558 & - & \\
\hline Redução de 6\% & 40.403 & - & 40.650 & - & 40.945 & - & Durovini et al. 11 \\
\hline Custo total $(\mathrm{R} \$) * *$ & 48.288 .145 & 33.515 .644 & 48.618 .165 & 33.720 .721 & 48.662 .971 & 33.964 .786 & \\
\hline Impacto orçamentário & \multicolumn{2}{|c|}{14.772 .501} & \multicolumn{2}{|c|}{14.897 .444} & \multicolumn{2}{|c|}{14.698 .185} & \\
\hline$\%$ do orçamento do PNCT & \multicolumn{2}{|c|}{7} & \multicolumn{2}{|c|}{7} & \multicolumn{2}{|c|}{8} & \\
\hline Aumento de $37 \%$ & 58.885 & - & 59.246 & - & 59.674 & - & Durovini et al. 11 \\
\hline Custo total $(\mathrm{R} \$) * *$ & 70.377 .403 & 16.400 .156 & 70.808 .031 & 16.500 .506 & 71.320 .528 & 16.619.934 & \\
\hline Impacto orçamentário & \multicolumn{2}{|c|}{53.977 .247} & \multicolumn{2}{|c|}{54.307 .525} & \multicolumn{2}{|c|}{54.700 .594} & \\
\hline$\%$ do orçamento do PNCT & \multicolumn{2}{|c|}{27} & \multicolumn{2}{|c|}{27} & \multicolumn{2}{|c|}{28} & \\
\hline \multicolumn{8}{|l|}{ Produção *** } \\
\hline $\begin{array}{l}2 \text { Xpert MTB/RIF para } 1 \text { caso } \\
\text { de TB notificado }\end{array}$ & 98.859 & 85.964 & 99.464 & 86.490 & 100.183 & 87.116 & Calculado \\
\hline Custo total $(\mathrm{R} \$) * *$ & 30.316 .476 & 20.588 .808 & 30.501 .974 & 20.714 .787 & 30.722 .742 & 20.864 .718 & Calculado \\
\hline Impacto orçamentário & \multicolumn{2}{|c|}{9.727 .665} & \multicolumn{2}{|c|}{9.787 .187} & \multicolumn{2}{|c|}{9.858 .025} & \\
\hline$\%$ do orçamento do PNCT & \multicolumn{2}{|c|}{5} & \multicolumn{2}{|c|}{5} & \multicolumn{2}{|c|}{5} & \\
\hline \multicolumn{8}{|l|}{ de TB notificado } \\
\hline Custo total $(\mathrm{R} \$) * \star$ & 92.918 .337 & 59.369 .317 & 93.486 .890 & 59.732 .589 & 94.163 .532 & 60.164 .923 & Calculado \\
\hline Impacto orçamentário & \multicolumn{2}{|c|}{33.549 .020} & \multicolumn{2}{|c|}{33.754 .301} & \multicolumn{2}{|c|}{33.998 .609} & \\
\hline$\%$ do orçamento do PNCT & \multicolumn{2}{|c|}{17} & \multicolumn{2}{|c|}{17} & \multicolumn{2}{|c|}{18} & \\
\hline
\end{tabular}

PNCT: Programa Nacional de Controle da Tuberculose.

* Variação apenas para o Xpert MTB/RIF;

** Refere-se ao custo total do diagnóstico e do tratamento e inclui a variação do preço do cartucho de 9,98 Dólares para os anos de: $2015: 1$ Dólar = 3,64 Reais, 2016: 1 Dólar = 3,66 Reais e 2017: 1 Dólar = 3,50 Reais;

*** Considera o resultado do aumento de 15\% na notificação de casos de tuberculose pelo Xpert MTB/RIF multiplicado por 2 testes (melhor cenário) e por 11 testes (pior cenário).

tica eficiente para a entrega de insumos e reagentes, e locais disponíveis e adequados para o armazenamento dos cartuchos 18 .

Em relação ao mix de utilização das duas tecnologias no SUS, até 2014 o padrão de diagnóstico era a baciloscopia para todos os sintomáticos respiratórios. A partir desse ano, esperava-se que a substituição completa da baciloscopia pelo Xpert MTB/RIF fosse gradual, mas levando-se em conta que o objetivo do teste era substituir duas baciloscopias diagnósticas, adotou-se como pressuposto que a partir de 2015 ele seria a tecnologia diagnóstica de rotina. O Xpert MTB/RIF é preconizado como teste diagnóstico e na sua versão atual não tem sido indicado para o acompanhamento do tratamento 30 , e a baciloscopia permanece no SUS como o teste para esta indicação em todos os casos. Nesse sentido, a taxa de difusão do Xpert MTB/RIF aqui considerada está restrita ao diagnóstico da TB, ainda que a tecnologia esteja sendo aplicada para outros fins como mencionado, o que é um fator importante a ser considerado quando se trata de ganhos de economias de escala.

Os cenários alternativos apontados na análise de sensibilidade demostraram que quando a nova tecnologia for extremamente acurada (incremento da taxa de notificação em 37\%) 11 haverá um substancial aumento do impacto orçamentário, dado que ela seria capaz de diagnosticar corretamente mais indivíduos e, portanto, o custo total do tratamento dos verdadeiros-positivos aumentaria. Ainda, a realização de uma grande quantidade de testes (11 testes) para confirmar um caso de TB é outro aspecto relevante que pode refletir a ineficiência do processo nos laboratórios, o que impacta no custo 
do diagnóstico, cujo principal direcionador de custo é o cartucho 24 . Nesse sentido, não é suficiente analisar o GeneXpert MTB/RIF somente através de análise de custo-efetividade, mas também como a eficácia, preconizada em ensaios clínicos como um desfecho fundamental na definição de diagnósticos, e a eficiência podem influenciar o impacto orçamentário da tecnologia para o PNCT. Ademais, cabe mencionar que há tecnologia disponível para a detecção da TB no Brasil além do Xpert MTB/ RIF e, embora não haja estimativa do impacto orçamentário, pressupõe que a oferta de outros testes também seja discutida para o controle da TB 31.

A presente análise apresenta algumas limitações. Estudos desta natureza apresentam um elevado grau de incerteza dos parâmetros e pressupostos incorporados 16. O método epidemiológico foi selecionado para estimar a população de referência, variável que imputa importante incerteza nas análises de impacto orçamentário. O método ainda permite avaliar: (i) as possíveis restrições de indicações à utilização da tecnologia que, no caso do Xpert MTB/RIF, refere-se ao desperdício de cartuchos, incluído no cálculo do custo 24; (ii) aspectos da doença que podem afetar o acesso, e aqui podemos considerar a taxa de abandono do tratamento, dada a melhora dos sintomas em um curto espaço de tempo, mas que não se associa à cura da TB; e (iii) potencial aumento de demanda induzida, que neste caso pôde ser observado pela variação da taxa de notificação de casos pelo Xpert MTB/RIF 11 e pela quantidade de testes realizada para a notificação de um caso. Nesse sentido, a escolha do método epidemiológico justificou-se pela sua possibilidade de auxiliar na estimativa de alguns pressupostos e de suas potencialidades para minimizar as limitações deste trabalho. A tentativa de modelar estatisticamente o número de casos notificados pela baciloscopia ao longo de uma série temporal teve como objetivo estimar a população de interesse para os cinco anos definidos no horizonte analítico. Embora seja presumido que o número de casos de TB varie em função do tempo de acordo com uma determinada linha de tendência, esta pode não ser verificada quando se consideram anos não pertencentes à série histórica para a qual se tem informação. Também como limitação, cita-se a adoção do pressuposto no cenário de referência de que seriam produzidos cinco testes para a detecção de um caso de TB, que é uma estimativa média a fim de auxiliar os cálculos desta análise de impacto orçamentário, dado que se desconhece na literatura um padrão da quantidade de testes diagnósticos necessários para a notificação de um caso de TB. A variação entre 2 e 11 testes refletiu a produção dos municípios com grande e pequeno volumes de testes Xpert MTB/RIF e com diferentes taxas de sintomáticos respiratórios e de diagnóstico de $\mathrm{TB}$ entre os sintomáticos investigados 27 . Os resultados para o pior cenário são preocupantes, pois o PNCT oferece treinamento para os profissionais de saúde e aponta para a necessidade de explorar questões de pesquisa relacionadas à curva de aprendizagem e à eficiência na utilização do GeneXpert MTB/RIF. Apesar desse intervalo elástico (2 e 11 testes), os dados apresentados nas Tabelas 2 e 3 permitem que se ajustem os valores do impacto orçamentário conforme o volume médio de consumo anual por município. O mesmo ajuste poderá ser feito com base na variação cambial observada em dados oficiais de agentes econômicos 26 e para o custo do diagnóstico com o Xpert MTB/RIF 15,24. Ainda, aplicamos um aumento de 15\% na notificação de casos conforme estudo recente da tecnologia no Brasil 11. Para minimizar a incerteza que esse resultado poderia trazer, adotou-se na análise de sensibilidade a variação do intervalo de confiança (-6\% a 37\%) 11 . Finalmente, deve-se considerar que os dados para calcular o percentual do impacto no orçamento do PNCT estão baseados na literatura divulgada pela OMS em dólares, o que demanda uma análise mais cuidadosa quando se realiza a conversão para reais.

No que se refere aos dados utilizados e o risco de vieses, utilizamos o SINAN para a estimativa da população de interesse por ser uma base usada pelo PNCT como referência para a política de controle da TB. O custo do diagnóstico e tratamento com o Xpert MTB/RIF para o SUS foi baseado em estudo prévio 15,24 que apontou como principais limitações as diferenças salariais dos recursos humanos entre os municípios brasileiros e a estrutura física dos laboratórios, que varia em termos de porte e localização, podendo alterar os custos administrativos a serem imputados. 


\section{Considerações finais}

A multiplicidade de fatores intrínsecos à incorporação do GeneXpert MTB/RIF no SUS indica que tomar uma decisão tendo como referência a eficácia e a efetividade da tecnologia é importante, mas não suficiente. No Brasil, dada a diversidade dos serviços de saúde em termos de acesso, recursos financeiros, humanos e de infraestrutura, a incorporação torna-se um quebra-cabeças em que cada peça deve ser combinada corretamente.

Na América Latina, o Xpert MTB/RIF tem se mostrado favorável na detecção da TB em pacientes coinfectados pelo HIV 32,33. A maioria do países do continente tem registro de compra do sistema GeneXpert MTB/RIF e todos fazem parte da lista da Foundation for Innovative New Diagnostics. Porém, há insuficiência de dados que possibilitem o real conhecimento do grau de incorporação e da taxa de difusão do teste nos países latino-americanos, destacadamente em termos do impacto no orçamento dos serviços de saúde.

A incorporação dessa nova tecnologia pressupõe uma dependência da variação cambial no contexto econômico de cada país, que pode afetar o orçamento dos programas de controle da TB no caso de apreciação do dólar. Dados epidemiológicos, populacionais e de custo aqui apresentados fornecem informações no médio prazo do impacto da adoção da tecnologia no orçamento anual destinado ao controle da TB. Apesar das limitações apontadas, nossa intenção foi a de auxiliar pesquisadores e gestores a identificarem os principais parâmetros e pressupostos, a fim de estimar o impacto orçamentário do GeneXpert MTB/RIF e, assim, apoiar os gestores brasileiros e dos países latino-americanos no planejamento e gestão na sua decisão de incorporação.

\section{Colaboradores}

M. F. T. Pinto contribuiu com o desenho do estudo, coleta de dados, análise e interpretação dos resultados, redação da versão inicial e aprovação da redação final. R. Steffen contribuiu com a análise e interpretação dos dados e aprovação da versão final. A. Entringer contribuiu com a coleta de dados, análise e interpretação dos resultados, aprovação da versão final. A. C. C. Costa e A. Trajman contribuíram com a análise e interpretação dos resultados, redação da versão inicial e aprovação da versão final.

\section{Agradecimentos}

Os autores agradecem o apoio financeiro do Projeto Inovação para o Controle da Tuberculose (INco-TB), parceria entre o Programa Nacional de Controle da Tuberculose e a Fundação Ataulpho de Paiva, com o financiamento da Fundação Bill e Melinda Gates.

\section{Conflito de interesses}

Os financiadores não influenciaram o conteúdo do presente estudo ou a decisão de publicá-lo. A responsabilidade pelo desenho, execução e opiniões aqui considerados é somente dos autores.

\section{Referências}

1. World Health Organization. Global tuberculosis report 2014. http://apps.who.int/iris/bitst ream/10665/137094/1/9789241564809_eng. pdf?ua =1 (acessado em 10/Nov/2015).

2. Pan American Health Organization. Tuberculosis in the Americas. Regional report 2013: epidemiology, control and financing. http:// www.paho.org/HQ/index.php?option $=\mathrm{com}_{-}$ docman\&task $=$ doc_view\&Itemid $=270 \&$ gid $=2$ 9807\&lang=pt (acessado em 24/Jun/2015).

3. Departamento de Vigilância Epidemiológica, Secretaria de Vigilância em Saúde, Ministério da Saúde. Manual de recomendações para o controle da tuberculose no Brasil. Brasília: Ministério da Saúde; 2011.

4. Steingart KR, Ng V, Henry M, Hopewell PC, Ramsay A, Cunningham J, et al. Sputum processing methods to improve the sensitivity of smear microscopy for tuberculosis: a systematic review. Lancet Infect Dis 2006; 6:664-74.

5. Uys PW, Warren RM, van Helden PD. A threshold value for the time delay to TB diagnosis. PLoS One 2007; 2:e757.

6. Boehme CC, Nicol MP, Nabeta P, Michael JS, Gotuzzo E, Tahirli R, et al. Feasibility, diagnostic accuracy, and effectiveness of decentralised use of the Xpert MTB/RIF test for diagnosis of tuberculosis and multidrug resistance: a multicentre implementation study. Lancet 2011; 377:1495-505.

7. Steingart KR, Sohn H, Schiller I, Kloda LA, Boehme CC, Pai M, et al. Xpert ${ }^{\circledR}$ MTB/RIF assay for pulmonary tuberculosis and rifampicin resistance in adults. Cochrane Database Syst Rev 2013; (1):CD009593. 
8. World Health Organization. Xpert MTB/RIF assay for the diagnosis of pulmonary and extrapulmonary TB in adults and children: policy update. Geneva: World Health Organization; 2014.

9. World Health Organization. WHO monitoring of Xpert MTB/RIF roll-out: orders of GeneXperts and Xpert MTB/RIF cartridges. http:// apps.who.int/tb/laboratory/xpertmap/ (acessado em 15/Maio/2015).

10. Ministério da Saúde. Portaria no 48, de 10 de setembro de 2013. Torna pública a decisão de incorporar o Teste Xpert MTB/RIF para diagnóstico de casos novos de tuberculose e detecção de resistência à rifampicina no Sistema Único de Saúde - SUS. Diário Oficial da União 2013; 11 set.

11. Durovni B, Saraceni V, van den Hof S, Trajman A, Cordeiro-Santos M, Cavalcante S, et al. Impact of replacing smear microscopy with Xpert MTB/RIF for diagnosing tuberculosis in Brazil: a stepped-wedge cluster-randomized trial. PLoS Med 2014; 11:e1001766.

12. Vassall A, van Kampen S, Sohn H, Michael JS, John KR, den Boon S, et al. Rapid diagnosis of tuberculosis with the Xpert MTB/RIF assay in high burden countries: a cost-effectiveness analysis. PLoS Med 2011; 8:e1001120.

13. Menzies NA, Cohen T, Lin HH, Murray M, Salomon JA. Population health impact and cost-effectiveness of tuberculosis diagnosis with Xpert MTB/RIF: a dynamic simulation and economic evaluation. PLoS Med 2012; 9:e1001347.

14. Choi HW, Miele K, Dowdy D, Shah M. Costeffectiveness of Xpert(R) MTB/RIF for diagnosing pulmonary tuberculosis in the United States. Int J Tuberc Lung Dis 2013; 17:1328-35.

15. Pinto MP, Steffen, R, Cobelens F, van den Hof S, Entringer A, Trajman A. Cost-effectiveness of the Xpert MTB/RIF assay for tuberculosis diagnosis in Brazil. Int $\mathrm{J}$ Tuberc Lung Dis 2016; 20:611-8.

16. Mauskopf JA, Sullivan SD, Annemans L, Caro J, Mullins CD, Nuijten M, et al. Principles of good practice for budget impact analysis: report of the ISPOR Task Force on good research practices - budget impact analysis. Value Health 2007; 10:336-47.

17. Cepheid. The new GeneXpert ${ }^{\circledR}$ System. New systems. Same game-changing performance. http://www.cepheid.com/administrator/components/com_productcatalog/library-files/9a a 5ec89400ea87a35857be6954a799c-ebaf57a f725aafcd310a9acbcd8d4d59-GeneXpertBrochure-0112-10.pdf.

18. Durovni B, Saraceni V, Cordeiro-Santos M, Cavalcante S, Soares E, Lourenco C, et al. Operational lessons drawn from pilot implementation of Xpert MTB/Rif in Brazil. Bull World Health Organ 2014; 92:613-7.

19. Trajman A, Durovni B, Saraceni V, Menezes A, Cordeiro-Santos M, Cobelens F, et al. Impact on patients' treatment outcomes of XpertMTB/RIF implementation for the diagnosis of tuberculosis: follow-up of a stepped-wedge randomized clinical trial. PLoS One 2015; 10:e0123252.
20. Secretaria de Vigilância em Saúde, Ministério da Saúde. Boletim Epidemiológico 2015; 46(9).

21. Secretaria de Ciência, Tecnologia e Insumos Estratégicos, Ministério da Saúde. Diretrizes metodológicas. Análise de impacto orçamentário: manual para o Sistema de Saúde do Brasil. Brasília: Ministério da Saúde; 2012. (Série A: Normas e Manuais Técnicos).

22. Dobson AJ. An introduction to generalized linear models. 2nd Ed. New York: Chapman \& Hall/CRC; 2002.

23. Secretaria de Vigilância em Saúde, Ministério da Saúde. Boletim Epidemiológico 2016; 47(13).

24. Pinto M, Entringer A, Steffen R, Trajman A. Análise de custos de um teste de amplificação de ácido nucleico para o diagnóstico da tuberculose pulmonar sob a perspectiva do Sistema Único de Saúde. J Bras Pneumol 2015; 41:536-8.

25. Foundation for Inovative New Diagnostics. Price for Xpert MTB/RIF and FIND country list. http://www.finddiagnostics.org/ about/what_we_do/successes/findnegotiated prices/xpert_mtb_rif.html (acessado em 15/ Set/2015).

26. Banco Central do Brasil. Focus - relatório de mercado. Brasília: Banco Central do Brasil; 2015.

27. Coordenação Geral do Programa Nacional de Controle da Tuberculose, Departamento de Vigilância das Doenças Transmissíveis, Secretaria de Vigilância em Saúde, Ministério da Saúde. Monitoramento da rede de teste rápido molecular para tuberculose. Casos novos e retratamentos. Brasília: Ministério da Saúde; 2015.

28. Brasil. Lei no 12.401 , de 28 de abril de 2011. Altera a Lei no 8.080, de 19 de setembro de 1990, para dispor sobre a assistência terapêutica e a incorporação de tecnologia em saúde no âmbito do Sistema Único de Saúde - SUS. Diário Oficial da União 2011; 29 abr.

29. Secretaria de Vigilância em Saúde, Ministério da Saúde. Boletim Epidemiológico 2015; 44(9).

30. Secretaria de Vigilância em Saúde, Ministério da Saúde. Nota Informativa 2014; (9).

31. Scherer LC, Sperhacke RD, Ruffino-Netto A, Rosseti ML, Vater C, Klatser P, et al. Cost-effectiveness analysis of PCR for the rapid diagnosis of pulmonary tuberculosis. BMC Infect Dis 2009; 9:216.

32. Balcells ME, Garcia P, Chanqueo L, Bahamondes L, Lasso M, Gallardo AM, et al. Rapid molecular detection of pulmonary tuberculosis in HIV-infected patients in Santiago, Chile. Int J Tuberc Lung Dis 2012; 16:1349-53.

33. Carriquiry G, Otero L, Gonzalez-Lagos E, Zamudio C, Sanchez E, Nabeta P, et al. A diagnostic accuracy study of Xpert(R)MTB/RIF in HIV-positive patients with high clinical suspicion of pulmonary tuberculosis in Lima, Peru. PLoS One 2012; 7:e44626. 


\section{Abstract}

The study aimed to estimate the budget impact of GeneXpert MTB/RIF for diagnosis of tuberculosis from the perspective of the Brazilian National Program for Tuberculosis Control, drawing on a static model using the epidemiological method, from 2013 to 2017. GeneXpert MTB/RIF was compared with two diagnostic sputum smear tests. The study used epidemiological, population, and cost data, exchange rates, and databases from the Brazilian Unified National Health System. Sensitivity analysis of scenarios was performed. Incorporation of GeneXpert MTB/RIF would cost BRL 147 million (roughly USD 45 million) in five years and would have an impact of 23 to $26 \%$ in the first two years and some $11 \%$ between 2015 and 2017. The results can support Brazilian and other Latin American health administrators in planning and managing the decision on incorporating the technology.

Tuberculosis; Polymerase Chain Reaction; Diagnosis; Costs and Cost Analysis

\section{Resumen}

El objetivo del estudio fue estimar el impacto presupuestario del GeneXpert MTB/RIF para el diagnóstico de la tuberculosis, desde la perspectiva del Programa Nacional de Control de la Tuberculosis de Brasil, valiéndose de un modelo estático, apoyado en el método epidemiológico entre 2013 y 2017. Se comparó un test Xpert MTB/RIF con dos baciloscopias diagnósticas. Se utilizaron datos epidemiológicos, poblacionales, de costes, la tasa de cambio y bases de datos del Sistema Único de Salud. Se realizó un análisis de sensibilidad por escenarios. La incorporación del GeneXpert MTB/ RIF demandaría un montante de R\$147 millones en cinco años y representaría un impacto de 23 a $26 \%$ durante los dos primeros años, y de cerca de un $11 \%$ entre 2015 y 2017. Los resultados pueden apoyar a los gestores brasileiros y de los países latinoamericanos en la planificación y gestión a la hora de decidir incorporar este tipo de tecnología.

Tuberculosis; Reacción en Cadena de la

Polimerasa; Diagnóstico; Costos y

Análisis de Costo
Recebido em 28/Dez/2015

Versão final reapresentada em 16/Nov/2016

Aprovado em 13/Dez/2016 